\title{
TERRITORIOS DE LA MOVILIDAD EN DISPUTA: CARTOGRAFÍAS CRÍTICAS PARA EL ANÁLISIS DE LAS MIGRACIONES Y LAS FRONTERAS EN EL ESPACIO SUDAMERICANO
}

\author{
Contested Territories of Mobility: Critical Cartographies for the \\ Migration and Borders Analysis in the South American Space
}

\author{
Lourdes Basualdo* \\ Eduardo Domenech* \\ Evangelina Pérez ${ }^{* * *}$
}

\begin{abstract}
Resumen. Este artículo explora ciertos usos de las "cartografías oficiales" y da cuenta de diversas experiencias de "cartografías heréticas" de la migración y las fronteras con la intención de contribuir a la reflexión sobre las diferentes formas de apropiación de las prácticas del mapeo para la investigación crítica y el activismo político. Así, el texto alienta el uso y la problematización de las cartografías y de las prácticas de mapeo de la migración y las fronteras para el análisis crítico de las disputas y luchas en torno al control y la libertad del movimiento en el espacio sudamericano.
\end{abstract}

Palabras clave: migraciones; fronteras; cartografía; mapeo colectivo; Sudamérica.

Abstract. This article explores certain uses of "official cartographies" and accounts for various experiences of migration and borders "heretical cartographies" with the aim of contributing to the reflection on different ways of appropriation of mapping practices for critical research and political activism. Thus, the text encourages the use and problematization of migration and borders cartographies and mapping practices for the critical analysis of disputes and struggles around the control and freedom of movement in the South American space.

Keywords: migrations; borders; cartography; collective mapping; South America.

Centro de Investigaciones y Estudios sobre Cultura y Sociedad (CONICET y UNC) y Centro de Estudios Avanzados (FCS y UNC). Córdoba, Argentina. E-mail: mlubasualdo@gmail.com. Orcid: 0000-0001-7489-296X.

** Consejo Nacional de Investigaciones Científicas y Técnicas (CONICET), Centro de Investigaciones y Estudios sobre Cultura y Sociedad (CONICET y UNC) y Centro de Estudios Avanzados (Facultad de Ciencias Sociales y UNC). Córdoba, Argentina. E-mail: eduardo.domenech@gmail.com. Orcid: 0000-0003-4182-5917.

${ }^{* * *}$ Centro de Investigaciones y Estudios sobre Cultura y Sociedad (CONICET y UNC) y Centro de Estudios Avanzados (FCS y UNC). Córdoba, Argentina. Orcid: 0000-0002-3449-3435. 
Este artículo forma parte de un esfuerzo colectivo más amplio que, con un sentido programático, busca profundizar la comprensión crítica sobre la configuración y transformación de las políticas y prácticas de control del movimiento y la emergencia de las luchas migrantes que el aumento y la diversificación de los controles fronterizos han hecho multiplicar en el espacio sudamericano. Ante el aumento cuantitativo de los movimientos migratorios y la aparición de nuevos contingentes de migración intra y extrarregional, expertos que transitan el mundo burocrático y académico, han augurado una nueva "crisis de gobernabilidad" de las migraciones. En este contexto, el conocimiento experto producido por gobiernos, organismos intergubernamentales regionales e internacionales y think tanks está adquiriendo cada vez mayor centralidad en las disputas en torno a la definición política de las migraciones y las fronteras. La producción de cartografías de los "flujos migratorios" se ha constituido en una de las nuevas manifestaciones del control de la movilidad humana. Por otra parte, las políticas de migración dominantes entran en tensión con las prácticas y estrategias de movilidad de los sujetos migrantes y sus reivindicaciones políticas. Las diferentes experiencias de "luchas migrantes" se han reconfigurado frente a la intensificación de los controles migratorios y fronterizos en el cambiante escenario político regional. El contramapeo se ha convertido en una nueva herramienta de lucha y de construcción de nuevos horizontes para la movilidad y el cruce fronterizo.

En este artículo exploramos ciertos usos de las "cartografías oficiales" y damos cuenta de diversas experiencias de "cartografías heréticas" de la migración y las fronteras con la intención de contribuir a la reflexión sobre las diversas formas de apropiación de las prácticas del mapeo para la investigación crítica y el activismo político. Debido al potencial heurístico de las cartografías críticas para ofrecer modos alternativos de representación y visibilización de las migraciones, así como de espacialización y visualización del control de los movimientos migratorios y las luchas migrantes, resulta sugerente profundizar la interrogación acerca del papel del conocimiento experto, específicamente la producción de datos y mapas, en el control del movimiento y las fronteras, como también de las ideas y modos de actuación que involucran a las cartografías dominantes y alternativas acerca del control migratorio y fronterizo en la región sudamericana. En este sentido, este artículo busca alentar el uso y la problematización de las cartografías y de las prácticas de mapeo de la migración y las fronteras para el análisis crítico de las disputas y luchas en torno al control y la libertad del movimiento en el espacio sudamericano.

Nuestro interés por la cartografía y las prácticas de mapeo surgió a partir de la búsqueda de nuevos recursos y materiales que permitieran renovar las herramientas teórico-prácticas de docencia e investigación, además de cuestionar los modos habituales de habitar y vincularnos con los diversos 
espacios y colectivos que conforman el heterogéneo universo del llamado "activismo migrante". En un primer momento incorporamos el contramapeo entre los contenidos a desarrollar en un seminario sobre migraciones destinado a estudiantes de licenciatura. En una segunda instancia, hicimos uso del "mapeo colectivo" para el desarrollo de un proyecto que tenía como propósito conocer y reflexionar sobre las experiencias cotidianas de migrantes y su relación con las políticas migratorias nacionales, así como avanzar en la discusión colectiva sobre alternativas para una política local en materia de migración. Finalmente, el conocimiento cartográfico dominante y las prácticas de contramapeo pasaron a formar parte de una propuesta de investigación orientada a indagar críticamente las transformaciones de las políticas de control migratorio y fronterizo en el espacio sudamericano. En este proceso convergieron preocupaciones individuales y grupales sobre la reflexión acerca de las luchas migrantes a partir de la mirada de la autonomía de las migraciones, la apropiación del concepto de régimen de migración y fronteras, la discusión latinoamericana sobre la categoría de territorio y la incorporación del análisis de lo visual como herramienta de investigación en el campo de las movilidades.

Este texto retoma reflexiones y experiencias de investigación y activismo de diferentes contextos locales o regionales que proponen una lectura crítica de las cartografías dominantes de la migración y las fronteras y que problematizan o se apropian del contramapeo o del mapeo colectivo como una herramienta de indagación científica y de acción política. Estos proyectos cartográficos alternativos suelen destacar el poder de las prácticas cartográficas no solo en la representación de los "flujos migratorios", sino también en el modo en que intervienen en la defensa de la libertad de movimiento como en su negación a través de prácticas de control (Casas-Cortes, Cobarrubias, 2007; Cobarrubias, 2009). Al mismo tiempo, este texto busca dialogar con distintas aproximaciones y desarrollos del pensamiento crítico como la reflexión procedente de aquellos estudios feministas que prestan atención a los procesos diferenciales de aceptación y rechazo de las/os migrantes a partir de lógicas de género y regímenes sexuales hegemónicos o dominantes en las prácticas de control de las movilidades (Luibhéid, Cantú, 2005; Luibhéid, 2006; Camacho Lucio, 2015). También las discusiones en torno a las nociones de "espacio" y "territorio" provenientes de la geografía crítica latinoamericana (Porto Gonçalvez, 2002; Mançano Fernandez, 2007; Haesbaert, 2013) inspira nuestra indagación crítica de las cartografías oficiales sobre las migraciones y las fronteras en tanto resaltan la dimensión política de los territorios, siempre vinculada a la existencia de disputas de poder por el control sobre el espacio, incluido el control de los "flujos" o "movimientos". Por último, el acercamiento a las imágenes cartográficas también se nutre de una concepción más amplia que busca prestar atención a "la diversidad de las subjetividades visuales que 
operan en cualquier 'mundo de imágenes'" (Poole, 2000, p. 31). Esto posibilita atender a los diferentes regímenes escópicos que cada época considera verosímil respecto a lo visible (Jay, 2003) y a los efectos concretos que el mapeo y la mirada cartográfica tienen sobre las formas a través de las cuales comprendemos y codificamos el mundo (Pickles, 2003).

La estructura del artículo establece dos partes que, al mismo tiempo, se corresponden con dos dimensiones de las cartografías críticas y constituyen la idea central del texto: el desarrollo de la cartografía crítica de las migraciones y las fronteras necesita tanto del análisis crítico de las representaciones visuales y prácticas cartográficas dominantes como de la producción de cartografías que desafíen la visión estatocéntrica inscripta en los modos de mapear las migraciones (lo visible y lo oculto) y recuperen los conflictos, las divisiones y las disputas de poder que las fronteras contienen, desde la perspectiva de quienes protagonizan los movimientos y los cruces. Así, la primera parte está dedicada a las "cartografías oficiales" de la migración y las fronteras y realiza una lectura exploratoria sobre sus fundamentos y efectos como prácticas de control migratorio y fronterizo, recuperando la noción de cartopolítica (Van Houtum, 2012), utilizada por trabajos empíricos recientes (Cobarrubias, 2019; Campos-Delgado, 2018). La segunda parte aborda las "cartografías heréticas", noción inspirada en el trabajo de Abdelmalek Sayad, para dar cuenta de nuevas modalidades y experiencias de mapeo, entendidas como prácticas de negociación, contestación y resistencia, surgidas en el marco de la intensificación de los controles migratorios y fronterizos, así como de las múltiples violencias ejercidas sobre los sujetos migrantes.

\section{Las cartografías oficiales de la migración y las fronteras: el análisis crítico de la "cartopolítica"}

Uno de los roles que históricamente ha desempeñado el mapa ha sido el de ofrecer una "imagen del orden espacial del mundo" (Pickles, 2003, p. 9). La forma de representación del espacio ofrecida por la cartografía ha operado como un dispositivo de control, en la medida que el saber cartográfico del mapa construido por los geógrafos, se constituyó, inicialmente, en un conocimiento explotable por las autoridades coloniales y, más adelante, pasó a formar parte del discurso del nacionalismo que permitió justificar la existencia de las fronteras (De Diego, 2008). A partir del siglo XVI, la confección de mapas precisos con la ubicación y los recursos de los "territorios descubiertos" en América, fue una estrategia fundamental para que las potencias europeas expandieran su poder colonial, de modo que la cartografía se convirtió en un saber geopolítico determinante, siendo luego una herramienta indispensable en el ámbito militar como también en la delimitación, establecimiento y sustentación de la soberanía de los Estado-nación (Montoya Arango, 2007). El 
saber cartográfico que estructuró históricamente el desarrollo de los mapas se erigió sobre el modelo visual regido por el perspectivismo cartesiano que se constituyó, según Martin Jay, en uno de los regímenes escópicos dominantes de Occidente en la era moderna. La construcción de la imagen sobre la posición dominante del perspectivismo se basó en la idea de que este modo de visión lograba transmitir la "experiencia natural" de la vista (Jay, 2003, p. 225). El distanciamiento, la organización de las cosas y la mirada "desde arriba", propios del perspectivismo, constituían los principios fundamentales que regían la construcción de los mapas a partir de la representación de territorios fijos (De Diego, 2008).

La imagen estática, fija y delimitada del territorio que muestran las cartografías oficiales invisibiliza las disputas de poder que ocurren en los territorios por el control del espacio, incluidas las estrategias para influir, afectar y controlar el movimiento y el acceso a ciertos territorios, ya sea de personas o mercancías (Sack, 1986). La reflexión sobre el "territorio", una contribución fundamental de la geografía crítica latinoamericana, remite indefectiblemente a su dimensión política, dada su vinculación a la existencia de disputas de poder por el control sobre el espacio, incluido el control de los "flujos" o "movimientos". Desde esta perspectiva, el territorio es concebido como un espacio de disputa de poder en el que conviven diferentes territorialidades atravesadas por las nociones de multidimensionalidad y multiescalaridad (Porto Gonçalvez, 2002; Mançano Fernández, 2007). A su vez, no hay territorios que no sean fruto de procesos de territorialización, es decir, la apropiación de un espacio (sea físico o simbólico) por parte de un grupo como condición para su existencia y reproducción. Es en los territorios donde se ponen de manifiesto distintos procesos de territorialización, lo cual genera tensiones, disputas y luchas.

La idea de territorio vinculada a los procesos de control del espacio propuesta por Sack, es retomada por Haesbaert, quien explica que el territorio se compone de redes que articulan distintos territorios a partir del movimiento y del tránsito de las personas y objetos, lo cual es clave para comprender un aspecto central del territorio: el control del espacio mediante el control de la movilidad (Haesbaert, 2013). Esta visión del territorio lleva a una concepción de poder vinculada directamente, por un lado, a quienes tienen el control de los flujos -pudiendo desencadenarlos, direccionarlos o limitarlos- y por el otro, a quienes no poseen ese control y sufren las tentativas de "inmovilización", aunque siempre de manera relativa. En este sentido, para Haesbaert, los procesos de contención territorial, "dibujan nuevas-viejas estrategias de control territorial", novedosas en sus formas contemporáneas pero antiguas en cuanto que los muros constituyen estrategias históricas de control. En tiempos de múltiples y complejas formas de in-movilidad, 
aquello que legitima las políticas de movilidad y las técnicas de seguridad y de contención territorial, es el supuesto de que hay sujetos y/o grupos cuya movilidad debe ser contenida y/o restringida. Sin embargo, estas políticas dan lugar a una multiplicidad de micropolíticas que representan formas muy diversas de reacción y/o resistencia a estos procesos de contención hegemónica (Haesbaert, 2015).

Las prácticas cartográficas forman parte de las políticas y regímenes de control de la movilidad. La noción de cartopolítica propuesta por Henk van Houtum (2012), recuperada recientemente para el análisis crítico de las cartografías de las políticas migratorias y fronterizas de la Unión Europea y México (Cobarrubias, 2019; Campos-Delgado, 2018), refiere a "las estrategias cartográficas diseñadas para afirmar el control sobre el territorio" (Lacy Bueno, Van Houtum, 2015, p. 484), lo cual supone "la imposición visual de control y significado sobre el espacio, así como sobre sus habitantes, su comportamiento e ideologías" (Lacy Bueno, Van Houtum, 2015, p. 485)ํ. Al combinar la política y los mapas, este neologismo remite a los modos dominantes de cartografíar la frontera, a las formas establecidas históricamente por el Estado para representarla. En este sentido, la cartopolítica da cuenta de la representación estatal de la frontera y, al mismo tiempo, (re)produce los modos convencionales o dominantes de visualizar las migraciones. Como productora del orden nacional, la cartopolítica es una manifestación concreta del pensamiento oficial. En este sentido, la cartopolítica podría ser comprendida como un producto y efecto del "pensamiento de Estado" (Bourdieu, 1997; Sayad, 2010), una forma de pensamiento "que refleja, a través de sus propias estructuras (estructuras mentales), las estructuras del Estado, así hechas cuerpo" (Sayad, 2010, p. 385). El pensamiento de Estado no se circunscribe al pensamiento del Estado: su producción acontece en múltiples espacios sociales y a través de la participación de una diversidad de actores de distinta naturaleza. A pesar de su circulación internacional, el conocimiento experto involucrado actualmente en la producción cartográfica de instituciones intergubernamentales o supranacionales está decididamente atravesado por el pensamiento de Estado que, a su vez, lo constituye como tal.

La representación de las migraciones y las fronteras en los mapas está íntimamente vinculada a la naturalización del Estado-nación en los esquemas de percepción, pensamiento y acción. A pesar de la intensificación de los movimientos internacionales, la frontera es representada habitualmente en los mapas oficiales como algo estático, intocable y fijo (Van Houtum, 2012). Esta visualización rígida de las fronteras es cuestionada por las "migraciones irregulares" (Van Houtum, 2012; Campos-Delgado, 2018), asociadas a la idea

1 Cabe destacar que el término "cartopolítica" (Van Houtum, 2012; Bueno Lacy, Van Houtum, 2015) fue advertido a partir de la lectura del trabajo de Cobarrubias (2019). 
de "desorden" en las narrativas actuales de control del movimiento como la "gobernanza global de la migración". En este sentido, las prácticas cartográficas oficiales apuntan a establecer límites y orden, es decir, son tanto prácticas de fronterización como de ordenamiento del espacio ${ }^{2}$. La narrativa estatal sobre la movilidad humana contemporánea plasmada en las cartografías oficiales asume, como plantean Tazzioli y Garelli, una espacialidad y temporalidad específicas. Por un lado, las migraciones aparecen en los mapas como una especie de desviación de la norma territorial a la vez que algunas prácticas de movimiento son "traducidas" mediante un proceso de abstracción en "flujos migratorios" y algunos sujetos son reificados como "migrantes" (Tazzioli, Garelli, 2019). Estos "flujos migratorios" suelen ser graficados con puntos, flechas y líneas de gran espesor, en particular si se trata de "migraciones irregulares", transmitiendo la idea de que son "flujos" masivos, unidireccionales e imparables (Van Houtum, 2012). Por otro lado, como indican Tazzioli y Garelli, los mapas oficiales de la migración, tanto los elaborados por los Estados como por las agencias internacionales, se sustentan en una temporalidad lineal oculta. "Capturadas" y enmarcadas como un incuestionable objeto de gobierno, las migraciones son cartografiadas habitualmente como un movimiento lineal de sur a norte (Tazzioli, Garelli, 2019). Los mapas oficiales dan cuenta de "rutas", no de "itinerarios", dos procesos espaciales diferentes: las "rutas" suponen control migratorio y los "itinerarios" equivalen a prácticas migrantes; mientras el primer concepto apunta a los modos de regulación destinados a canalizar o encauzar los movimientos, el segundo remite a los caminos y pasadizos de los migrantes cuya movilidad excede dichos intentos institucionales de control (Casas-Cortes et alii, 2015). Las cartografías oficiales no toman en cuenta la heterogeneidad de quienes se mueven ni de su posible movimiento circular (Van Houtum, 2012) o de sus marchas y contramarchas, sus estancias breves o prolongadas en algún lugar al cual llegaron de modo imprevisto o provisorio. El planteo que subyace a estos mapas es, como indica van Houtum (2012), que la migración es un movimiento lineal único que lleva a un destino final.

Estas representaciones cartográficas han empezado a experimentar algunas alteraciones a partir de la incorporación de nuevas herramientas de seguimiento y monitoreo de los movimientos de población y la aparición de nuevos actores internacionales en el campo de los controles migratorios y fronterizos. Algunos trabajos recientes de Maribel Casas-Cortes, Sebastián Cobarrubias y John Pickles constituyen iniciativas pioneras del análisis crítico de la cartopolítica producida por expertos en el terreno de las migraciones y las fronteras (Casas-Cortes, Cobarrubias, 2018; Casas-Cortes et alii, 2019;

2 Esta imbricada relación entre fronterización y ordenamiento queda expresado, en inglés, en el juego de palabras que permite el término b/ordering, cuyo uso ha tenido últimamente una enorme expansión. 
Cobarrubias, 2019). Esta producción aborda la política de externalización del control migratorio, desarrollada en la Unión Europea, mediante el análisis de las políticas del International Centre for Migration Policy Development (ICMPD), un think tank creado en 1993 con su sede principal en Viena y cuya actuación ha tenido una enorme influencia en la propagación del modelo del migration management y, más recientemente, en el desarrollo de un enfoque basado en las "rutas" de la migración. Los trabajos mencionados se centran en el I-Map, una herramienta de mapeo interactivo y base de datos que sigue la evolución de las rutas migratorias que atraviesan el Sahara, el Mediterráneo y Europa del Este en dirección a la Unión Europea, rastreando las rutas de la "migración irregular". Muestran que estas cartografías oficiales de las migraciones y las fronteras producidas por expertos no pretenden obtener una visión de territorialidad centrada en el Estado, sino una imagen que intenta capturar las rutas cambiantes de los cuerpos en movimiento (Cobarrubias, 2019). Como lo han señalado en otro lugar, el foco del control no está puesto en el paso fronterizo, sino en el movimiento a través de las fronteras (Casas-Cortes et alii, 2017). Esta frontera móvil es particularmente evidente en los modos en que se ejerce el control sobre los migrantes "irregulares" (Campos-Delgado, 2018). Las nuevas tecnologías y herramientas cartográficas interactivas empleadas para captar y representar la incertidumbre de los complejos y turbulentos flujos de migraciones (Casas-Cortes et alii, 2017; Cobarrubias, 2019) son posibles también a partir de transformaciones fundamentales en el régimen escópico actual, que registra un creciente asentamiento de la imagen electrónica que ya no cumple con la función de archivo de la imagen tradicional, es decir, no busca necesariamente recuperar el pasado, sino orientarse hacia el futuro y tiene lugar en un espacio de interconexión entre sujetos que se afectan mutuamente (Brea, 2007).

La "cartoexpertise" (Cobarrubias, 2019) ha penetrado también las prácticas y estructuras del régimen sudamericano de migración y fronteras. Las transformaciones acontecidas durante las dos últimas décadas en el campo de las políticas de migración y, en particular, la reconfiguración de las políticas y prácticas de control migratorio y fronterizo han puesto de relieve que la región no permanece al margen de los emergentes y adaptables esquemas y modelos internacionales de control del movimiento $y$, en especial, de las "migraciones irregulares" (Domenech, 2013). Como parte de la denominada data politics (Bigo et alii, 2019), el intercambio y producción de datos junto con la elaboración y uso de mapas de los movimientos migratorios, especialmente aquellos de gran magnitud o considerados como "forzados", para el seguimiento y detección de "amenazas" y "riesgos" se ha expandido en la región. La Matriz de Monitoreo del Desplazamiento (DTM por sus siglas en inglés) constituye una de las más recientes expresiones de 
la cartopolítica desarrollada por organismos internacionales con el apoyo de gobiernos nacionales en Sudamérica. Frente a la magnitud y la velocidad que adquirió la migración de nacionales venezolanos, en particular desde el 2015, la Organización Internacional para las Migraciones (OIM) buscó a través de diferentes medios conocer el perfil de las/os migrantes así como las características y dinámicas de las rutas migratorias. Hasta aquel momento, la OIM publicaba periódicamente informes institucionales de distinta naturaleza con datos obtenidos de fuentes secundarias principalmente. A partir del fuerte incremento que experimentó la emigración venezolana, la OIM comenzó a llevar adelante acciones de seguimiento y monitoreo de esta población, produciendo datos primarios mediante la aplicación de una "encuesta de monitoreo de flujos migratorios" (FMS, por sus siglas en inglés) bajo el fundamento de la promoción de "una migración segura, ordenada y regular".

En este contexto, la salida masiva de personas, definida como "crisis migratoria" por numerosos gobiernos nacionales y organismos intergubernamentales de carácter regional e internacional, favoreció un escenario propicio para que la OIM empezara a utilizar por primera vez en el contexto sudamericano la DTM. Según las definiciones oficiales, como se explica en diferentes lugares (páginas web, folletos, boletines, informes, etc.), la DTM es "un sistema para seguir y monitorear el desplazamiento y la movilidad de las poblaciones" y está diseñada para "capturar, procesar y proporcionar información crítica a los tomadores de decisiones y al personal de respuesta durante emergencias" como también para "contribuir a una mejor comprensión de los flujos migratorios". La DTM fue creada en 2004 con el fin de "monitorear el desplazamiento interno en Iraq". Luego fue adaptada para ser utilizada en contextos de conflicto, desastres naturales, "emergencias complejas" y "crisis prolongadas" de numerosos países, especialmente de la periferia mundial. Desde entonces la DTM de la OIM ha pasado a formar parte de diversos procedimientos y estrategias institucionales para la recolección, procesamiento y difusión de datos sobre grupos de población migrante y "flujos migratorios" en contextos de "crisis migratorias", convirtiéndose en una instancia de provisión de insumos para la producción de "cartografías de desplazamientos".

En Sudamérica, la herramienta empezó a ser aplicada en los países limítrofes o más próximos a Venezuela y ha estado dirigida a capturar específicamente la cantidad, la composición y la dinámica del "flujo migratorio" de nacionales venezolanos, identificado como una "población en situación de movilidad humana". En Colombia se realizó un primer proyecto piloto hacia finales del 2016 y luego se desarrolló una segunda fase a mediados del 2017; también se usó la DTM en la ciudad de Bogotá a finales del 2017. En Perú, se realizaron dos rondas de recolección de información en 
las zonas fronterizas con Ecuador y Chile entre finales del 2017 y comienzos del 2018. También en Guyana se implementó en el último mes del 2017. En Ecuador se realizó en dos puntos fronterizos y en la ciudad de Quito hacia mediados del 2018. En Brasil, hubo una primera ronda en Roraima a comienzos del 2018. Más recientemente, la DTM ha sido implementada en países sudamericanos más alejados y con menor presencia de nacionales venezolanos. En Argentina, se realizó una primera ronda a mediados de 2018 mediante el componente de Monitoreo de Flujo de la DTM en tres pasos fronterizos, financiada por la Oficina de Población, Refugiados y Migración (PRM por sus siglas en inglés) del gobierno de los Estados Unidos. A partir de la información obtenida de estas encuestas, se han confeccionado "mapas de rutas migratorias" de la población venezolana "en situación de movilidad" como parte de la estrategia de la DTM. En general, estos mapas muestran lugares de partida y de destino, puntos de ingreso o salida y de tránsito, circuitos o vías principales, y medios de transporte utilizados. Las "rutas migratorias" son representadas con puntos, líneas y flechas unidireccionales que apuntan a diversos países de destino. Estas líneas continuas o punteadas de colores, que pretenden dar cuenta además del tipo de transporte prioritariamente utilizado, se destacan sobre un fondo de tono claro, con límites fronterizos trazados de modo casi imperceptible, que denotan territorios nacionales cuyas fronteras son atravesadas. En estos mapas oficiales, el borramiento del ejercicio de los controles migratorios y fronterizos es total.

\section{Las cartografías heréticas de la migración y las fronteras: el contramapeo como disidencia}

Mediante la noción de "cartografías heréticas" de la migración y las fronteras, creada a partir de las contribuciones teóricas de Abdelmalek Sayad, pretendemos caracterizar aquellas propuestas e iniciativas de contramapeo que buscan subvertir la imaginación u orden cartográfico dominante. Estas prácticas de contramapeo pueden consistir en la reapropiación de los mapas convencionales y el análisis crítico de la cartopolítica o las cartografías oficiales, así como en la producción de mapas que problematizan los regímenes de migración y fronteras y cuestionan las prácticas de control y vigilancia del movimiento o que buscan dar cuenta de las "luchas migrantes" y las estrategias y experiencias cotidianas y subjetivas de quienes se enfrentan a los intentos institucionales por disciplinar la movilidad. Lo herético en Sayad (2010) supone una interrogación crítica de los preceptos del "pensamiento de Estado", una operación de impugnación o ruptura con las convenciones del orden nacional establecido, en definitiva, un acto de liberación y sublevación frente al orden social instituido. Por otra parte, en estos proyectos cartográficos disidentes, hay una búsqueda por llevar adelante una "geografía desde abajo", como propone 
Porto-Gonçalves (2015), prestando atención a los modos en que los grupos sociales "grafían" o "marcan" la tierra, en lugar de elaborar mapas para el gobernante.

La noción de contramapeo refiere a la posibilidad de elaborar mapas alternativos a las estructuras de poder dominantes (Hodgson, Schroeder, 2002) con el objetivo de recuperar miradas de los propios sujetos que transitan los territorios, sus luchas y resistencias, lo cual es invisibilizado en los mapas oficiales. A diferencia de la cartopolítica, uno de los propósitos de las cartografías críticas de la migración es "dar cuenta del movimiento en sus dimensiones cualitativas y sensibles, en particular desde la perspectiva de los que se desplazan" (Bacon et alii, 2016, p. 185). La interrogación de las representaciones oficiales desplegadas en las cartografías dominantes permite a los grupos y movimientos sociales analizar y transformar espacios ya existentes (Cobarrubias, 2009) y, en ese sentido, la cartografía crítica se vuelve un medio para (re)crear los territorios o para articular nuevas formas de habitar y subvertir la frontera (Casas-Cortes, Cobarrubias, 2007). Así, al cuestionar los estándares de representación, las diversas modalidades de cartografías críticas como el contramapeo "desafían el proyecto positivista de la neutralidad del conocimiento" (Bacon et alii, 2016, p. 201).

Diferentes proyectos de "mapeos activistas" (Cobarrubias, 2009) han emergido en el contexto europeo a partir de la puesta en circulación de las cartografías oficiales y de una consecuente reapropiación crítica de las herramientas y conocimientos contenidos en estos saberes estatales sobre la migración y las fronteras. Este es el caso del uso que recibe watchthemed.net, la plataforma de mapeo en línea que surgió vinculada al proyecto de investigación Oceanografía Forense, coordinado por Charles Heller y Lorenzo Pezzani desde Londres, Ginebra y Túnez (Casas-Cortes et alii, 2017). A modo de práctica de contravigilancia esta plataforma se alimenta de la información cartográfica generada por los propios Estados europeos para controlar los movimientos transnacionales mediante tecnologías de teledetección y produce información sobre la violación de los derechos humanos y/o las causas de muerte de migrantes que intentan cruzar el mar Mediterráneo, identifica situaciones en las que se encuentren en peligro, son detenidos, o no reciben asistencia estatal (Casas-Cortes et alii, 2017). Al mismo tiempo, la información producida por watchthemed.net es compartida con las/os migrantes y con grupos activistas, a través de Alarmphone, en la búsqueda de organizar o planificar los procesos migratorios de un modo más seguro.

El contramapeo también ha operado como una herramienta de denuncia al régimen fronterizo de la Unión Europea, por medio de múltiples intervenciones que impugnan las cartografías oficiales, entre las que se destacan, como se ha identificado y sistematizado en diferentes trabajos 
(Casas-Cortes, Cobarrubias, 2007; Pickles et alii, 2017; Bacon et alii, 2016; Casas-Cortes et alii, 2017), los cuadros del colectivo Anarchitektur, formado por arquitectos activistas ubicados en Alemania y Europa Occidental que muestran los procedimientos, pasos burocráticos y lugares por los que transitan las/os migrantes alemanes durante el "mundo tortuoso de la deportación"; Hackitectura, un mapa creado por activistas, artistas, hackers y arquitectos de Andalucía y el norte de Marruecos que recrea la frontera entre España y África del Norte para mostrar nuevas relaciones entre ambos lugares en lugar de aceptar la división geopolítica de la frontera, lo que conduce a recrear el territorio fronterizo como un espacio de flujos y conexiones (Casas-Cortes, Cobarrubias, 2007; Casas-Cortes et alii, 2017); la serie MigMap, un sistema virtual que permite visualizar procesos, lugares y actores que conforman el espacio social y discursivo del régimen fronterizo, a través de bases de datos, mapas, cartogramas, proporcionados por diferentes herramientas informáticas; y, por último, dos herramientas focalizadas en la visibilización y denuncia de los efectos de las políticas migratorias de la Unión Europea: los mapas fronterizos de Le Monde Diplomatique, que muestran las muertes de migrantes ocurridas en la Unión Europea desde 1993 a 2006, y las representaciones cartográficas del Atlas de la migración de Migreurope, que dan cuenta de diversas dimensiones del control migratorio y fronterizo.

Otras prácticas de contramapeo llevadas a cabo por migrantes han sido realizadas con la intención de reinscribir sus experiencias migratorias en nuevos mapas. En algunos casos, estas prácticas cartográficas han partido del análisis y la reapropiación crítica de la información contenida en los mapas oficiales acerca de sus trayectorias y/o sobre las formas en que son nominados o clasificados. Ejemplos de estas contracartografías se encuentran en los mapas elaborados en una serie de talleres colectivos iniciados hacia finales de 2011 con integrantes de la Red Sin Fronteras de Aragón, una asociación de inmigrantes de Zaragoza, España, y coordinados por Maribel Casas-Cortes y Sebastián Cobarrubias, a partir de los cuales las/os migrantes se opusieron a aceptar los supuestos contenidos en las cartografías oficiales construidas por el I-Map sobre sus experiencias migratorias y comenzaron a cuestionar las ideas "tergiversadas" en esos saberes expertos, mediante el trazado de nuevos itinerarios que permitiesen reflexionar sobre los mecanismos de control y gestión fronteriza que operaban en sus cuerpos (Casas-Cortes et alii, 2017). En una línea de trabajo similar, un proyecto de talleres de contracartografías titulado "Crossing Maps", impulsado por la geógrafa Sara Mekdjian y desarrollado entre artistas, investigadores, solicitantes de asilo y refugiadas/os en Grenoble, Francia, durante mayo y junio de 2013, buscó discutir las nociones centrales asociadas a las categorías estatales tales como "verdaderos o falsos refugiados", y las representaciones oficiales del espacio y el tiempo que marcan las rutas 
de las/os migrantes para comenzar a crear nuevos mapas comunes capaces de expresar otras experiencias migratorias (Mekdjian et alii, 2014).

En América Latina, desde los años noventa, existe una multiplicidad de experiencias de cartografía crítica proveniente de colectivos conformados por investigadoras/es militantes y activistas y se han desarrollado numerosos proyectos de mapeo bajo diferentes modalidades y denominaciones ("cartografía social", "mapeo colectivo", "mapeo participativo", etc.), aunque no todas sean identificadas necesariamente como "contramapeos". Estas iniciativas cartográficas han formado parte de luchas territoriales e identitarias de poblaciones y comunidades indígenas y campesinas fundamentalmente, además de haber sido promovidas por organizaciones ambientalistas ${ }^{3}$. En algunos casos, estos procesos de construcción de mapas han involucrado de diversas maneras a gobiernos, ONGs, organizaciones de cooperación internacional y organismos financieros regionales $o$ internacionales. Paulatinamente, la herramienta del mapeo también ha empezado a ser utilizada en las luchas urbanas por la vivienda y las acciones en contra de los desalojos gubernamentales y otros desplazamientos forzados, así como se ha incorporado a las movilizaciones en contra de los abusos policiales y represión de la protesta social. Asimismo, en distintas partes del continente, las luchas feministas se han apropiado del mapeo como método para visibilizar y denunciar las desigualdades y violencias basadas en las divisiones de género, etnia/raza y clase. Solo de modo reciente han surgido propuestas y experiencias de mapeo colectivo o contramapeo que involucran a grupos o colectivos migrantes y que tematizan sus demandas y reivindicaciones frente a las políticas estatales de distinta naturaleza.

Entre las diferentes propuestas de contramapeo originadas en el contexto latinoamericano, se destacan aquellas impulsadas en el marco de las luchas feministas por la defensa de los territorios en el contexto de reclamos ambientales y por la despenalización del aborto, además de las denuncias de feminicidios y de prácticas de criminalización del aborto. En estrecho diálogo con la geografía crítica latinoamericana y la geografía feminista, los colectivos Geografía Crítica de Ecuador y Miradas Críticas del Territorio desde el Feminismo (conformado por activistas de Ecuador, México, España, Brasil y Uruguay) han producido cartografías disidentes a partir de talleres de mapeo colectivo desde la noción de cuerpo-territorio para el abordaje de conflictos territoriales y ambientales desde una perspectiva de género y antipatriarcal. Por su parte, el colectivo Desbordes de Género ha producido mapas alternativos en la ciudad de Quito que cuestionan miradas tradicionales alrededor del

\footnotetext{
3 Véase Offen (2009) sobre el "mapeo indígena" en América Latina y Acselrad y Régis Coli (2010) sobre diversas experiencias de "mapeo participativo" en Brasil.
} 
género y la sexualidad. Desde estas coordenadas políticas, algunos de estos grupos de investigación militante han empezado a desarrollar proyectos de cartografía crítica centrados específicamente en las experiencias migrantes para ilustrar distintas expresiones de violencia en los sistemas fronterizos. El Colectivo de Geografía Crítica de Ecuador ha desarrollado mapeos sobre los principales nodos de violación de derechos de las personas migrantes venezolanas y sus experiencias de territorialización a partir de su migración por Sudamérica y cartografías que muestran "multiterritorialidades fragmentadas" en la provincia fronteriza de Esmeraldas, al noroeste del Ecuador, a causa de la violencia de Estado que ha producido desplazamientos de familias, allanamiento de territorios indígenas y asesinatos ${ }^{4}$.

En Argentina, desde el año 2008, el dúo Iconoclasistas lleva a cabo múltiples talleres de "mapeo colectivo" empleando diferentes tipos de soportes gráficos y visuales, con el objetivo de "construir colectivamente miradas territoriales que impulsan y facilitan prácticas colaborativas y de transformación" (Iconoclasistas, 2013, p. 5). Su trabajo se ha centrado en el acompañamiento a grupos militantes, organizaciones barriales y vecinales, movimientos sociales y artísticos en sus reivindicaciones socioambientales y las luchas por el acceso a la ciudad en múltiples ciudades de Argentina y en algunos países de la región y España. Sus talleres de mapeo colectivo y publicaciones también han sido fuente de inspiración para el colectivo Orangotango, un grupo de geógrafos alemanes críticos. Por otra parte, en el ámbito de la Universidad Nacional de Córdoba se desarrolló un proyecto que pretendió poner en diálogo el conocimiento académico producido sobre las políticas de migración con los saberes y experiencias de vida de los sujetos migrantes, bajo el propósito de construir políticas locales "desde abajo" en materia migratoria, favoreciendo la producción de miradas alternativas sobre la regulación de la migración. Inspirados en la propuesta de Iconoclasistas, se realizaron una serie de talleres de reflexión y discusión con diversos grupos u organizaciones de migrantes en los que se desplegó la herramienta del mapeo colectivo para explorar las problemáticas que afectan a las/os migrantes en su vida cotidiana. También en México, a partir de la propuesta de Iconoclasistas, Prieto Díaz $(2016,2017)$ ha utilizado el mapeo colectivo para la reconstrucción de rutas o "caminos" por los que migrantes indocumentados realizan su tránsito por México con el objetivo de llegar a los Estados Unidos. En su investigación propone los "mapeos migrantes" como una herramienta de intercambio y diálogo entre el saber académico y las personas migrantes

4 En relación a la violencia estatal de los controles fronterizos, algunas investigaciones también han buscado cartografiar las representaciones territoriales, las estrategias y los recursos de mujeres transgéneros en sus tránsitos corporales en la frontera amazónica de Leticia (Colombia), Tabatinga (Brasil) y Santa Rosa (Perú) (Camacho Lucio, 2015). 
para la construcción colectiva de herramientas de análisis y colaborar en la visibilización del conocimiento del territorio de quienes cruzan la frontera.

\section{Reflexiones finales}

En América Latina, las cartografías críticas han recibido diversos usos a lo largo de más de tres décadas. Las prácticas cartográficas de la migración y las fronteras se originan en espacios de activismo y experiencias de mapeo relacionadas con conflictos y luchas sociales comprometidas con los efectos de las estructuras de dominación, desigualdad y violencia. Las discusiones en torno a la relación entre territorio y género en los movimientos y redes de organizaciones de mujeres de distintos países han dado lugar a la emergencia de prácticas de mapeo que buscan visibilizar y denunciar la violencia de las fronteras. La crítica feminista se tornó crucial para empezar a "desandar los mapas" construyendo otras cartografías que recuperen las distintas experiencias de habitar y de transitar el espacio según el género (Zubia, López, 2015) frente a la construcción de los mapas hegemónicos. De esta manera, las cartografías críticas podrían contribuir a desestabilizar las concepciones heteronormativas reproducidas en la construcción de jerarquías de género, raciales, sexuales y de clase social inscriptas en las prácticas y políticas de control migratorio y fronterizo.

Por otra parte, en el campo de la investigación, la exploración y análisis crítico del conocimiento cartográfico dominante sobre la migración y las fronteras, así como la producción de mapeos críticos, han abierto nuevas posibilidades para capturar las regularidades y contingencias de las políticas y regímenes de control y vigilancia del movimiento y para producir nuevas espacialidades, temporalidades y territorialidades de la migración. En Sudamérica, estas cartografías heréticas se circunscriben, por el momento, a proyectos de mapeo colectivo que involucran diversas experiencias migrantes a escala local. A su vez, en el marco de procesos globales de control migratorio y fronterizo, el monitoreo de las "rutas migratorias" se ha convertido en una de las más sofisticadas prácticas de disciplinamiento de la movilidad y en uno de los objetos privilegiados del conocimiento experto producido por algunas agencias internacionales para los Estados. El uso de estas recientes cartografías oficiales en el espacio sudamericano aún requiere ser estudiado como cartopolítica para poder comprender las próximas formaciones de los territorios de la movilidad en disputa.

\section{Referencias bibliográficas}

ACSELRAD, Henri; RÉGIS COLI, Luis. Disputas territoriales y disputas cartográficas: el surgimiento de nuevos sujetos "cartografantes". Revista Internacional de Filosofía Política, n. 35, p. 63-86, 2010. 
BACON, Lucie; CLOCHARD, Oliver; HONORÉ, Thomas; LAMBERT, Nicolas; MEKDJIAN, Sarah; REKACEWICZ, Philippe. Cartographier les mouvements migratoires. Revue européenne des migrations internationales, v. 32, n. 3/4, 2016.

BIGO, Didier; ISIN, Engin; RUPPERT, Evelyn. Data Politics. Worlds, Subjects, Rights. Abingdon, Oxon/New York, NY: Routledge, 2019.

BOURDIEU, Pierre. Razones prácticas. Sobre la teoría de la acción. Barcelona: Anagrama, 1997.

BREA, José Luis. Cambio de régimen escópico: del inconsciente óptico a la e-imagen. Revista de Estudios Visuales, v. 2, n. 4, p. 145-163, 2007.

CAMACHO LUCIO, Lina Maritza. Trayectorias fronterizas de mujeres transgénero: identidad, cuerpo y territorio. (Tesis de Maestría en Ciencias Sociales con mención en Género y Desarrollo) Facultad Latinoamericana de Ciencias Sociales, Flacso, Ecuador, 2015.

CAMPOS-DELGADO, Amalia. Counter-mapping Migration: Irregular, Migrants' Stories Through Cognitive Mapping. Mobilities, v. 13, n. 4, p. 488-504, 2018.

CASAS-CORTES, Maribel; COBARRUBIAS, Sebastián. Drawing Escape Tunnels through borders. In: An Atlas of Radical Cartography, p. 51-66, 2007.

CASAS-CORTES, Maribel; COBARRUBIAS, Sebastián. It is Obvious from the Map! Disobeying the Production of Illegality beyond Borderlines. Movements. Journal for Critical Migration and Border Regime Studies, v. 4, n. 1, 2018.

CASAS-CORTES, Maribel; COBARRUBIAS, Sebastián; DE GENOVA, Nicholas; GARELLI, Glenda; GRAPPI, Giorgio; HELLER, Charles; HESS, Sabine; KASPAREK, Bernd; MEZZADRA, Sandro; NEILSON, Brett; PEANO, Irene; PEZZANI, Lorenzo; PICKLES, John; RAHOLA, Federico; RIEDNER, Lisa; SCHEEL, Stephan; TAZZIOLI, Martina. New Keywords: Migration and Borders. Cultural Studies, v. 29, n. 1, p. 55-87, 2015.

CASAS-CORTES, Maribel; COBARRUBIAS, Sebastián; HELLER, Charles; PEZZANI, Lorenzo. Clashing Cartographies, Migrating Maps: Mapping and the Politics of Mobility at the External Borders of E.U. ACME An International Journal for Critical Geographies, v. 16, n. 1, p. 1-33, 2017.

CASAS-CORTES, Maribel; COBARRUBIAS, Sebastián; PICKLES, John. B/ordering Turbulence Beyond Europe: Expert Knowledge in the Management of Human Mobility. In: LINHARD, Tabea; PARSONS, Timothy H. (eds.). Mapping Migration, Identity, and Space, 2019, p. 257-281.

COBARRUBIAS, Sebastián. Mapping Illegality: The i-Map and the Cartopolitics of 'Migration Management' at a Distance. Antipode, v. 1, n. 25, p. 1-25, 2019.

COBARRUBIAS, Sebastián. Mapping Machines. Activist Cartographies of the Border and Labor Lands of Europe. Tesis doctoral, 2009.

COBARRUBIAS, Sebastián; PICKLES, John. The turn to cartographies and mapping practices in contemporary social movements. In: BARNEY WARF, Santa Arias (ed.). The Spatial Turn: Interdisciplinary Perspectives. Routledge, 2009, p. 36-58. 
COLECTIVO GEOGRAFÍA CRÍTICA DE ECUADOR. Geografías errantes en la exposición sobre la migración venezolana. Disponible en: <https:// geografiacriticaecuador.org/2018/11/09/geografias-errantes-en-la-exposicionsobre-la-migracion-venezolana/>. Acceso en: 06.08.2019.

COLECTIVO GEOGRAFÍA CRÍTICA DE ECUADOR. Violencias en evidencia. Cartografía de frontera en Ecuador. Disponible en: <https:// geografiacriticaecuador.org/2018/05/08/violencias-en-evidencia-cartografia-defrontera/>. Acceso en: 06.08.2019.

DE DIEGO, Estrella. Exposiciones coloniales y otras formas de representación. Contra el mapa. Madrid: Siruela, 2008.

DOMENECH, Eduardo. Las migraciones son como el agua: Hacia la instauración de políticas de control con rostro humano. La gobernabilidad migratoria en la Argentina. Polis. Revista Latinoamericana, n. 35, p. 119-142, 2013.

HAESBAERT, Rogerio. Del mito de la desterritorialización a la multiterritorialidad. Cultura y representaciones sociales, v. 8, n. 15, 2013.

HAESBAERT, Rogerio. Sobre as i-mobilidades do nosso tempo (e das nossas ciudades). Mercator, v. 14, n. 4, p. 83-92, 2015.

HODGSON, Dorothy; SCHROEDER, Richard. Dilemmas of Counter-mapping Community Resources in Tanzania. Development and Change, v. 33, n. 1, p. 79100, 2002.

ICONOCLASISTAS. Manual de mapeo colectivo: recursos cartográficos críticos para procesos territoriales de creación colaborativa. Buenos Aires: Tinta Limón, 2013. JAY, Martin. Regímenes escópicos de la modernidad. Buenos Aires: Paidós, 2003.

LACY BUENO, Rodrigo; VAN HOUTUM, Henk. Lies, Damned Lies \& Maps: The EU's Cartopolitical Invention of Europe. Journal of Contemporary European Studies, v. 23, n. 4, p. 477-499, 2015.

LUIBHÉID, Eithne. Sexual Regimes and Migration Controls: Reproducing the Irish Nation-State in Transnational Contexts. Feminist Review, v. 83, n. 1, p. 60-78, 2006.

LUBHÉID, Eithne; CANTU, Lionel (eds.). Queer Migrations: Sexuality, U.S. Citizenship, and Border Crossings. Minneapolis: University of Minnesota, 2005.

MANÇANO FERNANDES, Bernardo. Territorios en disputa: campesinos y agribusiness. São Paulo: UNES, 2007.

MEKDJIAN, Sarah; AMILHAT-SZARY, Anne-Laure; MOREAU, Marie; NASRUDDIN, Gladeema; DEME, Mabeye; HOUBEY, Lauriane; GUILLEMIN, Coralie. Figurer les entre-deux migratoires Pratiques cartographiques expérimentales entre chercheurs, artistes et voyageurs. Carnets de géographes, n. 7, 2014.

MONTOYA ARANGO, Vladimir. El mapa de lo invisible. Silencios y gramática de poder en la cartografía. Universitas Humanística, n. 63, p. 155-179, 2007.

OFFEN, Karl. O mapeas o te mapean: mapeo indígena y negro en América Latina. Tabula Rasa, n. 10, p. 163-189, 2009. 
PICKLES, John. A History of Space. Cartographic Reason, Mapping and the GeoCoded World. Routledge, 2003.

PICKLES, John; COBARRUBIAS, Sebastián; CASAS-CORTES, Maribel. Le regard cartographique, Les nouvelles cartographies des frontières, et les responsabilités du cartographe. Géoesthétique, 2017.

POOLE, Deborah. Visión, Raza y Modernidad. Una economía visual del mundo andino de imágenes. Lima: Casa de Estudios del Socialismo, 2000.

PORTO GONÇALVEZ, Carlos Walter. Da geografia às geo-grafias. Um mundo em busca de novas territorialidades. Cuadernos de Trabajo Instituto de Investigaciones Histórico-Sociales, n. 10, 2002.

PORTO GONÇALVEZ, Carlos Walter. Por una geografía desde abajo. Conferencia inaugural del V Congreso de Geografía de Universidades Públicas realizado entre el 23 al 26 de septiembre de 2015 organizado por el Departamento de Geografía de la Universidad Nacional del Comahue, Neuquén, Argentina, 2015.

PRIETO DÍAZ, Sergio. Cartografías de la subalternidad migratoria: Bestialización, inhumanidad y contrahegemonía en el contexto del plan frontera sur de México. (Tesis de doctorado en Ciencias Sociales y Políticas) Ciudad de México: Universidad Iberoamericana, 2017.

PRIETO DÍAZ, Sergio. La migración indocumentada desde una otra perspectiva: colonialidad, sujeto subalterno y mapeos migrantes. Iberóforum, v. 11, n. 22, p. 31-63, 2016.

SACK, Robert. Human Territoriality: Its Theory and History. Cambridge: Cambridge University Press, 1986.

SAYAD, Abdelmalek. La doble ausencia. De las ilusiones del emigrado a los padecimientos del inmigrado. Barcelona: Anthropos, 2010.

TAZZIOLI, Martina; GARELLI, Glenda. Counter-mapping, Refugees and Asylum Borders. In: MITCHELL, Katharyne; JONES, Reece; FLURI, Jennifer L. (eds.). Handbook on Critical Geographies of Migration. Cheltenham: Edward Elgar, 2019.

VAN HOUTUM, Henk. Remapping Borders. In: WILSON, Thomas; DONNAN, Hastings (eds.). A Companion to Border Studies. Boston, Massachusetts: Wiley Blackwell, 2012.

ZUBIA, Gonzalo Federico; LÓPEZ, Andrea Noelia. Geografía(s) feminista(s): itinerarios y debates por las reflexiones en torno al estudio cultural de las espacialidades. Question, v. 1, n. 46, p. 243-259, 2015. 